\title{
Electron Impact Ionization in the Presence of a Laser Field:
}

\section{A kinematically Complete $(\mathrm{n} \gamma \mathrm{e}, 2 \mathrm{e})$ Experiment}

\author{
C. Höhr, A. Dorn, B. Najjari, D. Fischer, C.D. Schröter, and J. Ullrich \\ Max-Planck-Institut für Kernphysik, \\ Saupfercheckweg 1, D-69117 Heidelberg, Germany
}

(Dated: November 4, 2004)

\begin{abstract}
Single ionization of He by $1 \mathrm{keV}$ electron impact in the presence of an intense $\left(\mathrm{I}=4 \times 10^{12} \mathrm{~W} / \mathrm{cm}^{2}\right)$ laser field $(\lambda=1064 \mathrm{~nm})$ has been explored in a kinematically complete experiment using a "Reaction Microscope". Distinct differences in the singly to fully differential cross sections compared to the field-free situation are observed which cannot be explained by a first-order quantum calculation. Major features, such as the number of photons exchanged and the modification of the energy spectrum of emitted electrons can be qualitatively understood within a simple classical picture.
\end{abstract}


The investigation of collisions in the presence of a strong electromagnetic field was first addressed theoretically more than half a century ago, when cross sections for "multiquantum Bremsstrahlung and absorption" were derived for the case of elastic scattering (see e.g. [1]). The topic has attracted continuous and increasing attention over the years (see e.g. [2] for a review) culminating in a series of recent theoretical papers where the motivation was several-fold:

First, field-assisted electron-impact excitation or ionization have now been demonstrated to be the basic underlying mechanisms for nonsequential multiple ionization in strong laser fields (see e.g. [3-5]). Here, a tunnelled electron, driven by the field recollides with its parent ion (for a review see [6]) thereby enhancing the multiple ionization yields by several orders of magnitude compared to uncorrelated, sequential tunnelling processes. Despite its paramount importance, the dynamics of the field-assisted electron recollision (i.e. the momentum exchange between the two active electrons, which strongly differ from the field-free behaviour) are far from being understood. Secondly, an intense laser-field was shown to considerably modify sub-femtosecond electron transfer processes in slow ion-atom collisions (see e.g. [7]) supporting hopes for ultra-fast electronic quantum control with possible applications in laser-driven fusion, plasma heating or the development and understanding of ultra-fast optoelectronic devices. Thirdly, the dynamical situation per se is most appealing from a fundamental point of view. Thus it has been predicted, for example, that thousands of low-energy photons might be exchanged with even a quite weak laser field during hard collisions of a fast ion with a target electron, illustrating the extremely effective coupling between the radiation field and collision-accelerated charged particles [8].

Experimentally, multiphoton emission and absorption occurring during elastic electronatom collisions in the presence of a $\mathrm{CO}_{2}$ laser field was first demonstrated in 1977 [9] and found to be in qualitative agreement with theoretical models based on the soft-photon "KrollWatson approximation" (KWA [10]). In this model the laser projectile interaction is treated to all orders, while the laser target interaction is neglected. Severe (order-of-magnitude) discrepancies to the KWA prediction found more recently (see e.g. [11]) under certain geometrical conditions have initiated a series of sophisticated treatments using the impulse approximation, a full Floquet [12] or a coupled-channel approach [13]. Only the last of the three found a qualitative confirmation of the experimentally observed slow variation of the cross sections with the number of exchanged photons, still however, being off by more than 
two orders of magnitude on absolute scale.

In 1987 simultaneous electron-photon excitation of helium was experimentally observed for the first time [14] in inelastic laser-assisted electron-atom collisions, which was likewise theoretically addressed well before (e.g. [15]). More recently an experiment was performed at higher laser frequencies [16] and theory was developed beyond a perturbative treatment [17-19].

In this letter we report on the first realization of an electron impact ionization experiment in the presence of a strong laser field, where the momenta of both outgoing electrons are determined allowing investigation of their respective emission characteristics. Using multi-particle imaging techniques ("Reaction Microscopes" [20, 21]) under laser-on/off but otherwise exactly identical experimental conditions, we clearly prove significant modifications of singly up to fully differential cross sections by the exchange of up to approximately 10 photons from a Nd:YAG laser field. The experimental results are compared to the predictions of a calculation in the first-order Born-approximation (FBA) without considering the interaction of the laser field with the target atom ("dressing") [22] and clear deviations are observed.

One major motivation for our experiments was to provide laser-assisted (e,2e) data under well-controlled experimental conditions in order to help clarify the puzzling discrepancies between experiment and theory observed in the momentum sharing of both electrons for strong-field double ionization. Moreover, exploring the interplay of attosecond dynamics, realized in the encounter with a collision time on the order of $\Delta \mathrm{t}=\mathrm{b} / \mathrm{v}=10^{-18} \mathrm{~s}$ (b impact parameter, $\mathrm{v}$ projectile electron velocity) with the femtosecond optical cycle time-scale of the laser, represents a generic case of ultra-fast quantum dynamics.

The experiment was performed at the Max-Planck-Institut für Kernphysik by overlapping a $1 \mathrm{keV}$ pulsed electron beam $(20 \mathrm{~Hz}, 1 \mathrm{~ns})$ and a Nd:YAG laser beam (10 Hz, $7 \mathrm{~ns}, 1064 \mathrm{~nm}$, $3 \mathrm{~J}$ ) at the position of a supersonic helium target beam (1 mm diameter, $10^{12}$ atoms $/ \mathrm{cm}^{3}$ ) at a base pressure of $10^{-8}$ Torr (Fig. 1). Care was taken, that the electron beam with a diameter of $140 \mu \mathrm{m}$ was completely embedded in space and time within the laser pulse which had a non-Gaussian approximately flat intensity distribution over a diameter of 100 $\mu \mathrm{m}$ (intensity: $\mathrm{I}=4 \times 10^{12} \mathrm{~W} / \mathrm{cm}^{2}$ ). Since the electron beam repetition rate exceeded that of the laser by a factor of two, field-free $(\mathrm{e}, 2 \mathrm{e})$ cross sections were measured simultaneously under exactly identical experimental conditions. Even though our Reaction-Microscope 


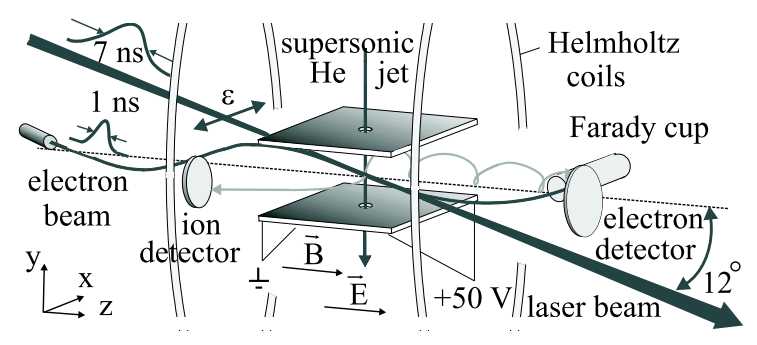

FIG. 1: Reaction-Microscope. The electron beam hits the target after a full-turn cyclotron cycle before propagating to the off-axis Faraday cup. The laser beam overlaps the electron beam colinearly in the interaction volume.

records the complete momentum vectors of emitted target electrons and recoil-ions with a solid angle of close to $4 \pi$, resulting in an immense time saving, the experiment had to run continuously over a period of approximately two months due to the low repetition rate of the highest-performance, commercially available high-power laser.

In the Reaction Microscope the low-energy emitted He electron "b" (exchange with the projectile electron "a" can be safely neglected at high energies) and the recoiling $\mathrm{He}^{+}$target ion "R" are projected by homogeneous electric $(2.5 \mathrm{~V} / \mathrm{cm})$ and magnetic (9.7 Gauss) fields upon position and time sensitive micro-channel plate detectors. The ion and electron longitudinal momentum components $\left(\mathrm{k}_{\|}=\mathrm{k}_{z}\right)$, along the $\mathrm{z}$-axis in Figure 1, are deduced from the times-of-flight (TOF), respectively. The two respective transverse momentum components $\left(\mathrm{k}_{x}, \mathrm{k}_{y}\right.$, with $\left.\mathrm{k}_{\perp}=\left(\mathrm{k}_{x}^{2}+\mathrm{k}_{y}^{2}\right)^{1 / 2}\right)$ are obtained from the impact positions on the detectors in the $x y$-plane and the TOFs (for details see [20, 21]). Thus, measuring the momentum vectors of both target fragments $\left(\overrightarrow{\mathrm{k}}_{b}, \overrightarrow{\mathrm{k}}_{R}\right)$ in coincidence in a kinematically complete experiment allows one to deduce the momentum of the scattered electron $\overrightarrow{\mathrm{k}}_{a}^{\prime}=\overrightarrow{\mathrm{k}}_{a}-\overrightarrow{\mathrm{k}}_{b}-\overrightarrow{\mathrm{k}}_{R}\left(\overrightarrow{\mathrm{k}}_{a}\right.$ : projectile electron initial mometum) as well as the momentum transfer $\overrightarrow{\mathrm{q}}=\overrightarrow{\mathrm{k}}_{a}-\overrightarrow{\mathrm{k}}_{a}=\overrightarrow{\mathrm{k}}_{b}+\overrightarrow{\mathrm{k}}_{R}$ occuring during the collision with $\overrightarrow{\mathrm{q}}=\left(\mathrm{q}_{\|}, \overrightarrow{\mathrm{q}}_{\perp}\right)$. Under present conditions, all target electrons with longitudinal momenta $\mathrm{k}_{b \|}>-1.4$ a.u. (-z-direction) and transverse momenta of $\mathrm{k}_{b \perp}<1.1$ a.u. are recorded simultaneously with resolutions $\Delta \mathrm{k}_{b \|}, \Delta \mathrm{k}_{b \perp}<0.08$ a.u.. (atomic units, a.u., are used throughout with $\mathrm{e}=\mathrm{m}=\hbar=1$; e, m: electron charge and mass, $\hbar$ : Planck's constant). The transverse electron momentum resolution depends on the TOF and the given value is an average. The $\mathrm{He}^{+}$ion acceptance was $\mathrm{k}_{R \|}<116$ a.u. and $\mathrm{k}_{R \perp}<4$ a.u. with resolutions along the various coordinates of $\Delta \mathrm{k}_{R \|}<0.12$ a.u., $\Delta \mathrm{k}_{R x}<0.13$ a.u. and $\Delta \mathrm{k}_{R y}<0.25$ a.u., the last of these being limited by the inherent $0.83 \mathrm{~K}$ temperature of the supersonic jet 
along the expansion direction.

Two different laser-induced reactions were observed. Firstly, the electron beam might excite the helium target to various states in a primary step (1) which are then transferred to the continuum by the absorption of one or more photons from the laser in a second step $(2)$ :

$$
\begin{aligned}
1 \mathrm{keV} \mathrm{e}^{-}+\mathrm{He} \rightarrow \mathrm{e}^{-}+\mathrm{He}^{*} & \\
& \mathrm{He}^{*}+\mathrm{n} \gamma \rightarrow \mathrm{He}^{+}+\mathrm{e}^{-} .
\end{aligned}
$$

By absorption of up to $\mathrm{n}=3$ photons $(\omega=0.043$ a.u. $\hat{=} 1.17 \mathrm{eV})$ from the different Stark shifted excited states (1s np), this leads to an increased yield of low-energy electrons with $\mathrm{E}_{b}<0.11$ a.u. $(\hat{=} 3 \mathrm{eV})$ beyond which no significant contribution could be observed. This electron-impact excitation photo-absorption ionization reaction is very interesting by itself and will be investigated in a subsequent paper. Secondly, the direct $(\mathrm{e}, 2 \mathrm{e})$ ionization reaction modified by the simultaneous absorption or emission of $\mathrm{n}$ photons, i.e. the (n $\gamma \mathrm{e}, 2 \mathrm{e})$ process, and the subject of the present paper, is observed:

$$
1 \mathrm{keV} \mathrm{e}^{-}+\mathrm{He} \pm \mathrm{n} \gamma \rightarrow 2 \mathrm{e}^{-}+\mathrm{He}^{+}
$$

In order to discriminate from the first reaction, all following spectra are recorded under the condition that the slow electron energy $\mathrm{E}_{b}>0.11$ a.u..

In principle, the number of exchanged photons can be determined in our kinematically complete experiment from the measured momenta of all target fragments, yielding the total inelasticity Q' of the reaction

$$
\mathrm{Q}^{\prime} / \mathrm{v}=\mathrm{Q} / \mathrm{v} \pm \mathrm{n} \gamma / \mathrm{v}=\mathrm{k}_{R \|}+\mathrm{k}_{b \|}-\mathrm{E}_{b} / \mathrm{v}
$$

(v: projectile velocity, $\mathrm{Q}=\mathrm{E}_{f}-\mathrm{E}_{i}$, with $\mathrm{E}_{f, i}$ the final and initial projectile energy, respectively). For single ionization without photon exchange $(n=0)$, this is just the ionization potential Q' $=\mathrm{Q}=\mathrm{IP}=0.904$ a.u. $\hat{=} 24.59 \mathrm{eV}$. Due to the jet-temperature limited recoilion momentum resolution and the large projectile velocity of 8.57 a.u., the overall Q'-value resolution is restricted to about 1.1 a.u., not sufficient for the discrimination of single photon exchanges with $\Delta Q^{\prime}=0.043$ a.u.. Nevertheless, distinct information on the number of exchanged photons " $\mathrm{n}$ " as well as on their probability distribution can be extracted from the present experiment. 


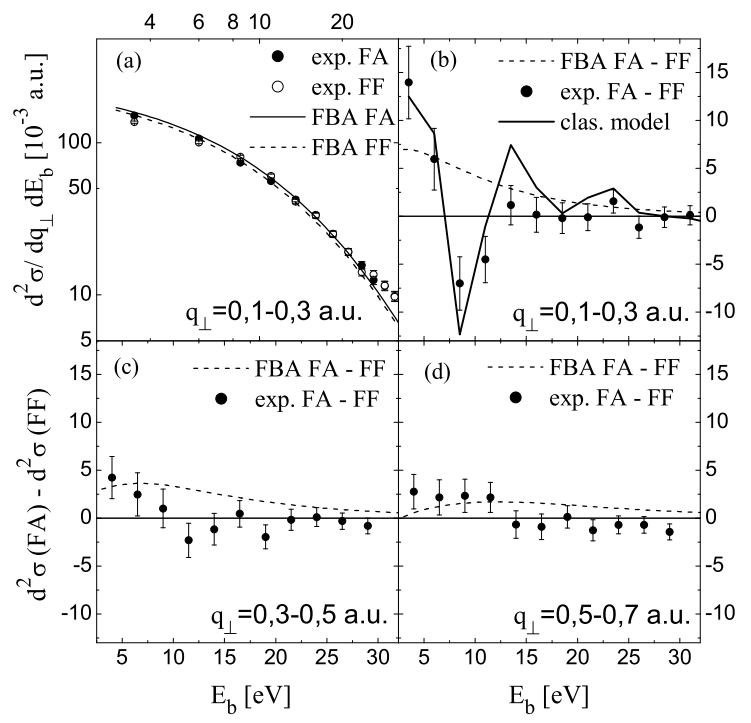

FIG. 2: Double differential cross sections $d^{2} \sigma / d \mathrm{q}_{\perp} d \mathrm{E}_{b}$ as a function of the electron energy $\mathrm{E}_{b}$ and different transverse momentum transfers $q_{\perp}$. (b-d): difference field-assisted (FA) minus field-free (FF) double differential cross section. The experimental data in (a) are scaled by a common factor to fit the overall size of the theory.

In Fig. 2 experimental and theoretical doubly differential electron emission spectra $d^{2} \sigma / d \mathrm{q}_{\perp} d \mathrm{E}_{b}$ (Fig. 2a) are shown along with differences of such spectra for laser on/off i.e. field-assisted (FA) and field-free $(\mathrm{FF})$ conditions for different transverse momentum transfers $q_{\perp}=\left|\vec{q}_{\perp}\right|$ by the scattered electron (Fig. 2b,c,d). Whereas nearly no effect due to the presence of the laser can be observed in the electron spectra alone (Fig. 2a), distinct patterns are found in the experimental as well as theoretical difference spectra: At low momentum transfers (Fig. 2b) an oscillatory behaviour of the experimental difference occurs which becomes less pronounced with increasing $q_{\perp}$ merging into just a slight enhancement of the laser-assisted cross section for low-energy electron emission $\left(0.11\right.$ a.u. $(3 \mathrm{eV})<\mathrm{E}_{b}<$ 0.55 a.u. $(15 \mathrm{eV}))$ at the largest $\mathrm{q}_{\perp}$ in Fig. 2 d. Within the experimental error bars, rough agreement between experimental results and theoretical predictions is found at large momentum transfers, whereas distinct differences occur for smaller $q_{\perp}$. This is in qualitative agreement with recent findings for elastic scattering in the presence of a laser field where deviations between experiment and theories were observed for small scattering angles of the projectile electron, i.e. for small momentum transfers ([11-13]).

Within the present theoretical model, described in detail in a forthcoming paper, the 
interaction of the projectile with the laser field is treated exactly to all orders by using an incoming and outgoing electron Volkov state. The projectile-target interaction is taken into account within the FBA, in the sense that the interaction of the projectile and the target is described via the exchange of one virtual photon, which is sufficient for a projectile velocity of 8.57 a.u.. The final state neglects the electron-electron as well as the projectile$\mathrm{He}^{+}$interactions. The slow emitted electron is described by a Coulomb-Volkov state. This includes the interaction of the slow electron with the residual ion but also with the laser field. Moreover, the initial target state is taken to be unperturbed by the laser-field, i.e. so-called "dressing" or dynamical polarization are not accounted for.

We have tried to explain our results and extract the number distribution of exchanged photons by a simple classical consideration. An electron being emitted into the continuum of a classical electric field oscillating with a frequency of $\omega$ will gain additional energy depending on its initial emission energy $\mathrm{E}_{b}^{i}$, its ponderomotive potential, i.e. its mean quiver energy in the oscillating field $\mathrm{U}_{P}=2 \pi \mathrm{I} / \mathrm{c} \omega^{2}$ with $\mathrm{I}=6.2 \times 10^{-4}$ a.u. the intensity of the laser field, c velocity of light, and depending on the phase $\omega t_{0}$ in the field when it was ejected in the collision. The final energy $\mathrm{E}_{b}$ for an angle $\theta$ between the momentum vector $\vec{k}_{b}$ and the polarization of the field is

$$
\mathrm{E}_{b} \approx \mathrm{E}_{b}^{i}-\sqrt{8 \mathrm{E}_{b} \mathrm{U}_{P}} \sin \left(\omega t_{0}\right) \cos \theta+2 \mathrm{U}_{P} \sin ^{2}\left(\omega t_{0}\right)
$$

If the electron is fast enough and leaves the laser focus within a time shorter than the laser pulse duration (which is the case for all energies considered here) it is accelerated by the ponderomotive potential gradient and gains the additional kinetic energy $\mathrm{U}_{P}$. In this model, the energy exchanged between the laser field and the projectile is neglected. On the basis of the Kroll-Watson model this is justified for small projectile scattering angles which dominate for single ionization (Fig. 2b). The maximum number of exchanged photons would then simply be $\mathrm{n}^{\max }= \pm\left(\mathrm{E}_{b}-\mathrm{E}_{b}^{i}\right) / \hbar \omega$. Averaging over all phases, taking the measured FF emitted electron energy distribution and folding it with field induced broadening due to photon absorption yields a modified electron spectrum. The difference of this spectrum minus the FF energy distribution (solid line in Fig. 2b) is found to be in best agreement with the measured value for an assumed laser intensity of $5.44 \times 10^{-4}$ a.u. $\hat{=} 3.5 \times 10^{12} \mathrm{~W} / \mathrm{cm}^{2}$ slightly lower than the experimentally determined result but well within the error bars. Moreover, the distribution of the number of exchanged photons obtained with this simple 


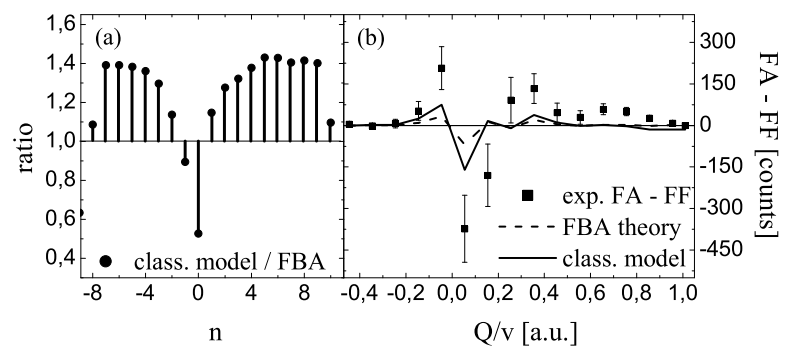

FIG. 3: (a) Ratio of the classical model/FBA photon distributions (b) difference FA - FF Q-value spectra for $\mathrm{q}_{\perp}<0.5$ a.u..

model is considerably broader than the one calculated within the FBA. This is illustrated in Fig. 3a, where the ratio of the classical model to the FBA photon distribution is shown. That is again in accordance with the previously mentioned elastic scattering results for small scattering angles, where the experimental photon number distribution is much broader than predicted.

As described before, we can get direct information on the number of exchanged photons by examining the Q'-value of the reaction. Subtracting the FF Q-value distribution from the FA one (Fig. 3b) shows an oscillatory behaviour resulting from a distinct broadening of the FA spectrum. Taking the number distribution extracted from our simple estimate to fit the electron energy distribution, folding it with the FF Q-value spectrum and subtracting the FF-spectrum yields the full line in Fig. 3b, being in reasonably good agreement with the data. Instead, taking the number distribution of exchanged photons from our FBAcalculation yields a considerably worse agreement (broken line in Fig. 3b) demonstrating again the enhanced probability of exchanging larger numbers of photons.

Finally we present triply differential cross sections TDCS $=d^{3} \sigma / 2 \pi k_{b} d \Omega_{b} d \mathrm{E}_{b} d q_{\perp}$ in Fig. 4 in the so-called coplanar geometry (the target electron is emitted into the scattering plane) for different momentum transfers q and emitted electron energies as indicated in the figure. For FA as well as FF conditions, one finds a distinct maximum along the momentum transfer direction, called the "binary-peak", pointing mainly towards $90^{\circ}$, representing target electrons being emitted as a result of a binary collision with the projectile. At small ejected electron energies and momentum transfers the so-called "recoil-peak" emerges, which might be interpreted as a binary electron being rescattered off the nucleus. For all emission energies and momentum transfers, the measured data are in fair agreement with the prediction 


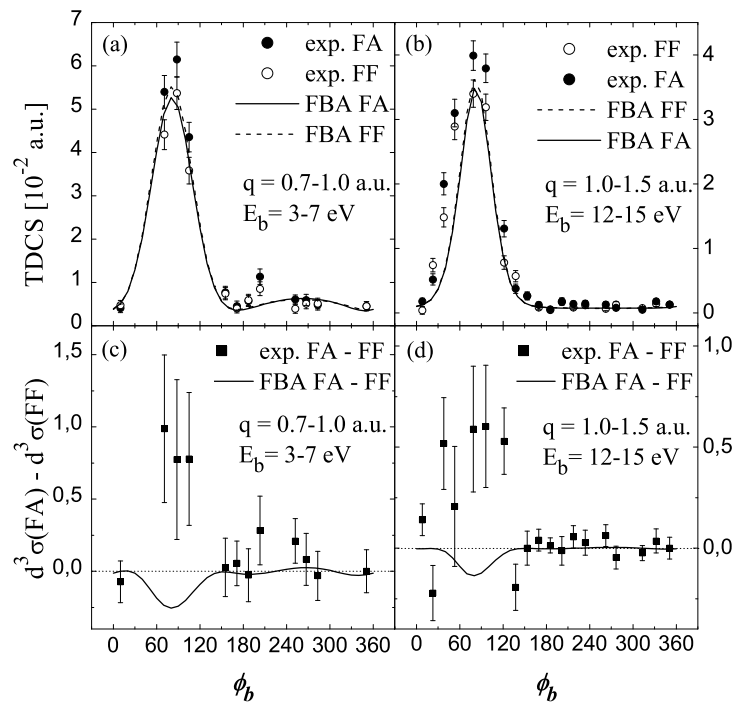

FIG. 4: Triple differential cross sections (TDCS) as a function of the emission angle $\phi_{b}$ of the ejected electron in the scattering plane with an opening angle of $\pm 20^{\circ}$.

of the FBA as it is expected at large projectile energies.

Deviations between FA and FF cross sections are observed in the difference FA-FF in Fig. 4c,d. Surprisingly, (and in disagreement with early calculations [23] as well as with the present predictions) the binary peak is enhanced under most of the dynamical conditions explored. Whereas the magnitude of the effect seems to be reasonably described by the calculations, the effect itself, i.e. enhancement or diminution of the peak just seems to be reversed! It would be interesting to compare our experimental data with the results of more refined non-perturbative calculations taking target polarization or dressing of the initial state into account [24-28].

In summary, we have presented first experimental laser-assisted kinematically complete (n $\gamma \mathrm{e}, 2 \mathrm{e})$ measurements and have compared them with the results of an FBA calculation. Distinct differences in doubly to triply differential cross sections between laser on/off conditions are observed which are poorly described by the first-order theory. A simple classical consideration to estimate the number distribution of exchanged photons showed this distribution to be considerably broader than predicted. This finding is in qualitative agreement with recent experimental results on field-assisted elastic scattering at small momentum transfers as well as with calculations going beyond a first order treatment.

In the future, we expect considerably enhanced resolution at lower electron impact ener- 
gies such that different channels due to different numbers of exchanged photons will become distinguishable. Such data will be of indispensable importance for the understanding of the recollision dynamics in strong-field non-sequential double ionization, where the maximum

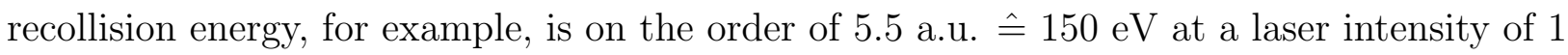
$\mathrm{PW} / \mathrm{cm}^{2}$ and certainly challenge theory.

We acknowledge support by the Leibniz-Programm of the Deutsche Forschungsgemeinschaft, from the MPG and the GSI. We thank A. Voitkiv for fruitful discussions.

[1] F.V. Bunkin, M.V. Fedorov, Sov. Phys. JETP 22, 844 (1966); see references therein.

[2] F. Ehlotzky et al., Phys. Rep. 297, 63 (1998).

[3] Th. Weber et al., Phys. Rev. Lett. 84, 443 (2000).

[4] R. Moshammer et al., Phys. Rev. Lett. 84, 447 (2000).

[5] A. Rudenko et al., Phys. Rev. Lett., submitted.

[6] R. Dörner et al., Adv. At. Mol. Phys. 48, 1 (2002).

[7] T. Kirchner, Phys. Rev. A 69, 063412 (2004).

[8] A. B. Voitkiv, J. Ullrich, J. Phys. B 34, 1673 (2001).

[9] A. Weingartshofer et al., Phys. Rev. Lett. 39, 269 (1977).

[10] N. M. Kroll, K. M. Watson, Phys. Rev. A 8, 804 (1973).

[11] B. Wallbank, J. K. Holmes, Phys. Rev. A 48, R2515 (1993), Can. J. Phys. 79, 1237 (2001).

[12] L. W. Garland et al., J. Phys. B 35, 2861 (2002).

[13] S. Geltman, J. Phys. B 35, 4787 (2002).

[14] N. J. Mason, W. R. Newell, J. Phys. B 20, L323 (1987).

[15] N.K. Rahman, F.H.M. Faisal, J. Phys. B 9, L275 (1976).

[16] S. Luan et al., J. Phys. B 24, 3241 (1991).

[17] A. Makhoute et al., J. Phys. B 35, 957 (2002).

[18] S. Vučić, Phys. Rev. A 51, 4754 (1995).

[19] P. Francken, C.J. Joachain, Phys. Rev. A 41, 3770 (1990).

[20] R. Moshammer et al., Nucl. Inst. Meth. B108, 425 (1996).

[21] J. Ullrich, Rep. Prog. Phys. 66, 1463 (2003).

[22] In the treatment of the laser-assisted (e,2e) collisions the laser field is coupled to the colliding 
system through the incident, scattered and ejected "free" electron.

[23] P. Cavaliere et al., Phys. Rev. A 24, 910 (1981).

[24] C. J. Joachain et al., Phys. Rev. Lett. 61, 165 (1988).

[25] P. Martin et al., Phys. Rev. A 39, 6178 (1989).

[26] A. Makhoute et al., J. Phys. B 32, 3255 (1999).

[27] M. Bouzidi et al., J. Phys. B 34, 737 (2001).

[28] S-M. Li et al., J. Phys. B 35, 557 (2002). 